\title{
When do leotards get their spots? Semantic activation of lexical neighbors in visual word recognition
}

\author{
JENNIFER M. RODD \\ University of Cambridge, Cambridge, England
}

\begin{abstract}
Shadowing and priming studies have provided strong evidence that during spoken word recognition, the meanings of different words that share their onset (e.g., captain and captive) are activated in parallel. In contrast, for visual word recognition, there is little evidence that the meanings of visually similar words are activated in parallel. This is consistent with the idea that for reading (in contrast to listening), since all the sensory information necessary to identify a word is available at once, any competition between visually similar words is resolved before their meanings are retrieved. However, Forster and Hector (2002) have recently shown that for nonwords (e.g., turple), some aspect of the meanings of lexical neighbors (e.g., turtle) can be activated. However, this finding is limited to nonwords. The activation of turtle's meaning in response to turple may occur because turple has no meaning. In normal reading, we do not encounter nonwords, and there is strong pressure on the reading system to produce meaningful representations for every word (even misspelled words). The two semantic categorization experiments reported here extend this finding to real words. Participants are slower to decide that leotard is not an animal because of its animal neighbor leopard. This shows that information about a word's meaning can be available before it has been uniquely recognized.
\end{abstract}

In the experiments reported here, the relationship between the recognition of individual words and the retrieval of their meanings was examined. Some models of word recognition assume that these processes are completely independent and that a word's meaning is retrieved only after its form has been uniquely identified. For example, in Forster's serial search model (e.g., Forster, 1976), words are identified by searching through the entries in the lexicon. Only after the correct access file has been identified is the semantic information accessed from a separate master file. In contrast, other models of both spoken and written word recognition assume that activation is cascaded, so that semantic representations begin to be activated before there is resolution at the level of form (e.g., Balota, Ferraro, \& Connor, 1991; Coltheart, Rastle, Perry, Ziegler, \& Langdon, 2001; Gaskell \& Marslen-Wilson, 1997; Plaut, McClelland, Seidenberg, $\&$ Patterson, 1996). For example, when we hear the word onset capt ..., we may start to retrieve the meanings of both captain and captive before there is sufficient input

This work was supported by a Research Fellowship from Peterhouse, Cambridge to J.M.R. and an MRC program grant to L. K. Tyler. Thanks to L. K. Tyler, H. E. Moss, and M. H. Davis for comments on an earlier draft of this article. Correspondence concerning this article should be addressed to J. M. Rodd, Department of Psychology, University College London, 26 Bedford Way, London WC1H 0AP, England (e-mail: jrodd@ ucl.ac.uk). to determine which word is present. Forster and Hector (2002) suggested an intermediate position between strictly form-first models and cascaded models. They proposed a form-first model in which lexical entries contain some semantic information. In particular, they suggested that lexical entries contain "associative links to semantic fields," where these links "may serve as indicators of possible category membership but do not provide detailed semantic information" (p. 1115). Therefore, on this view, although semantic representations are retrieved only once a lexical entry has been uniquely identified, lexical entries contain some semantic information.

In summary, models of word recognition make different claims about when semantic information is retrieved: Strictly form-first models assume that a word's meaning is retrieved only after its form has been uniquely identified, whereas many other models assume that there is earlier activation of meaning. This discrepancy has important implications for an issue that remains highly controversial: Can information about words' meanings influence the recognition of their forms? Autonomous models assume that semantic information cannot interact with earlier form-based recognition (e.g., Forster, 1981), whereas other authors have argued that feedback from semantics can influence how quickly words are recognized (e.g., Tyler, Voice, \& Moss, 2000). The question of whether word meanings are accessed in parallel is critical for this 
issue, because models that incorporate feedback from semantics to form-based representations must assume that word meanings begin to be activated before their forms have been uniquely identified. If word meanings are retrieved only once recognition is complete, feedback would provide little benefit, because the word would already have been identified. In contrast, if the meanings of several words are accessed in parallel, information about their meanings would be available sufficiently early to influence recognition. Therefore, this question of whether word meanings are accessed prior to their unique identification is important not only because it allows us to discriminate between form-first models and those that assume cascaded activation of semantics, but also because it has implications for the feedback debate.

In the case of spoken word recognition, there is strong evidence that word meanings are accessed in parallel. Marslen-Wilson $(1973,1985)$ has shown that some listeners can repeat connected speech at delays of only $250 \mathrm{msec}$ and that, even at these short latencies, their errors are syntactically and semantically constrained. This shows that listeners can retrieve semantic properties of words very early, often before words can be uniquely identified. Further evidence comes from cross-modal priming experiments in which participants hear a spoken prime and then make a lexical decision to a visual target. Marslen-Wilson (1987) and Zwitserlood (1989) have shown that when the visual target is presented before the auditory prime (e.g., captain) can be uniquely identified and is ambiguous between, for example, captain and captive, lexical decisions are faster to targets that are semantically related either to the prime or to its competitor. If, however, the target is presented at the end of the auditory prime (when it can be uniquely identified), there is facilitation only for words that are related to the prime itself. This shows that the meanings of the competing words are accessed before a word can be uniquely identified.

Evidence that this semantic information can influence the identification of word forms comes from Tyler et al. (2000), who investigated the effect of a semantic variable (imageability) on auditory lexical decision and word repetition. For both tasks, response times were shorter for high-imageability words than for low-imageability words. Crucially, for word repetition, they also manipulated the cohort size of the words (the number of words that share the word's onset). They found that the imageability effect was present only for words that have many cohort competitors. This interaction between imageability and cohort size suggests that semantic information affects lexical competition; if the locus of the imageability effect were postaccess, it would affect all words equally, irrespective of their cohort size. This result raises problems for models in which word recognition is autonomous from semantics but is consistent with models in which there is parallel activation of word meanings and in which the activation from these semantic representations feeds back to influence the identification of the word's form. Tyler et al. (2000) argued that semantic information is partic- ularly important for words with many competitors, because for these words the discrimination of the target word is particularly difficult.

Therefore, in the auditory domain, there is good evidence that word meanings are accessed before words are uniquely identified and that this semantic information can influence recognition. In contrast, despite the recent popularity of models that assume cascaded activation of semantics, there is little evidence that this is the case for written words. One crucial difference between print and speech is that speech develops over time. For example, when the word captain is heard, there exists a period in time (before its uniqueness point) when it is ambiguous between captain and captive, and the listener must wait for the disambiguating information. During this period in time, activating the meanings of the candidate words and using semantic constraints to decide which alternative is more likely could improve recognition. In contrast, in reading, most words are fixated on only once, so that the visual information about a word's form is potentially available all at once. At the point in time at which the visual input can generate a set of candidate words, there is enough information to identify which word is actually present. It is, therefore, plausible that there might be little benefit from retrieving semantic information before form-based recognition is complete. Of course, some visual features may be processed more quickly than others, and these differences might create a time window during which the semantic representations of a set of candidate words might be activated. However, this time window is likely to be smaller than it is for spoken words.

Despite this argument that the parallel activation of word meanings may be less likely for reading than for listening, one recent study has suggested that the meanings of visually similar words may be activated in parallel. Forster and Hector (2002) conducted a set of experiments designed to investigate the role of lexical neighbors in different tasks. In one of these experiments, participants performed semantic categorization (animal vs. nonanimal) on a set of items in which some of the nonanimals were nonwords. The participants were slower to reject nonwords that were neighbors of animals (e.g., turple, which has the neighbor turtle) than nonwords with no animal neighbors (e.g., tabric, which has the neighbor fabric). Therefore, some aspect of turtle's meaning must have been activated in response to turple, so that it interfered with the task. Therefore, semantic information must have been retrieved before the competition between the word forms was complete; if this were not the case, the meaning of turtle (which does not win the competition) would never be activated. Forster and Hector suggested that the semantic information that is retrieved is not part of the full semantic representation for that word but consists of semantic fields within the lexical entry that "serve as indicators of possible category membership but do not provide detailed semantic information" (p. 1115).

One limitation of this finding is that it is restricted to nonwords. The activation of turtle's meaning in response to turple may occur because turple has no meaning. In 
normal reading, we usually do not encounter nonwords, and there is strong pressure on the reading system to produce meaningful interpretations for every word (even misspelled words). Therefore, we may partially retrieve turtle's meaning because it is the best plausible interpretation. The crucial question is whether the meanings of neighbors are activated for real words. In the following experiments, this question was addressed by investigating whether participants would be slower to decide that, for example, leotard is not an animal because leopard's meaning is partially activated.

\section{EXPERIMENTS 1A AND 1B}

The task in Experiment 1A was to decide whether words were animal names. The crucial contrast was between two sets of words that were not animals. The experimental items (e.g., leotard) all had an animal neighbor (e.g., leopard). The control items were words (e.g., cellar) whose only neighbor was not an animal (e.g., collar). Neighbors are defined as words that differ from the target word by one letter, in any position (Coltheart, Davelaar, Jonasson, \& Besner, 1977). Unfortunately, it is impossible to ensure that these sets of words are perfectly matched. Forster and Hector (2002) noted that the similarity between words and their neighbors can vary. For example, the consonant change between leopard and leotard may be less salient than the vowel change between collar and cellar. Although such differences are unlikely to be consistent across the set of items, a control experiment was conducted to ensure that any observed difference was a real effect of neighbor type. In Experiment $1 \mathrm{~B}$, the same set of experimental and control stimuli were used as that in Experiment 1A, but the animal category exemplars were replaced with plant names, and the participants were asked to decide whether words were plant names. Because animal neighbors should not interfere with plant decisions, an interaction between task (experiment) and neighbor type was predicted, so that the experimental condition should differ from the control condition in Experiment 1A, but not in Experiment $1 \mathrm{~B}$.

\section{Method}

Participants. The participants were 34 University of Cambridge students (18 for Experiment 1A, 16 for Experiment 1B), who were paid for their time and had English as their first language and normal or corrected-to-normal vision.
Materials and Design. Eighty-seven words were used in each experiment (see the Appendix). In Experiment 1A, the 29 animal category exemplars included mammals, birds, insects, amphibians, fish, and reptiles; in Experiment 1B, the 29 plant category exemplars included trees, flowers, fruit, and vegetables. These sets were matched for length and word lemma frequency (CELEX lexical database; Baayen, Piepenbrock, \& Gulikers, 1995; see Table 1).

The experimental and control items were the same in both experiments. There were 29 experimental words that had an animal neighbor (e.g., leotard). Twenty-one of these items had no other neighbors; the remaining 8 items also had a second neighbor. The 29 control words (e.g., cellar) had only one neighbor, which was not an animal (e.g., collar). The experimental and control items were matched for lemma frequency and length. The neighbors of these two groups (which were never seen in the experiment) did differ slightly on frequency: The animal neighbors were slightly less frequent than the nonanimal neighbors. However, these small differences in the number and frequency of neighbors should have affected both experiments and could not produce the predicted interaction between task and condition.

Procedure. The practice block consisted of 27 words ( 9 category exemplars). The experiment began with five warm-up items, not included in the analysis. During the experiment, the participants saw the words in different random orders. Each trial began with a fixation cross that appeared for $500 \mathrm{msec}$. This was replaced by a word, which stayed on the screen until the participant responded. If the participant did not respond within 2,000 msec, the trial ended. After a delay of $565 \mathrm{msec}$, the next trial began. The stimuli were presented using the DMDX stimulus presentation program (Forster \& Forster, 2003).

\section{Results}

Mean response times and error rates were calculated separately across participants and items (see Figure 1 for item means). All correct responses were included in the response time analysis. Trials in which the participants did not respond within $2,000 \mathrm{msec}$ were classified as errors. The error proportions were arcsine transformed (Winer, Brown, \& Michels, 1991) to accentuate differences between low error rates.

Experiment 1A. Response times showed the predicted effect of neighbor type: Responses to the experimental words (e.g., leotard) were $72 \mathrm{msec}$ slower than those to the control words (e.g., cellar). This was significant in both participants and items analyses $\left[t_{1}(17)=\right.$ $\left.8.8, p<.001 ; t_{2}(56)=4.0, p<.001\right]$. Similarly, significantly more errors were made for the experimental words $\left[t_{1}(17)=2.1, p<.05 ; t_{2}(56)=2.3, p<.05\right]$.

Experiment 1B. Response times were $15 \mathrm{msec}$ longer for the experimental words than for the control words. This difference was significant in the participants analysis but was not even marginal in the items analysis $\left[t_{1}(15)=\right.$

Table 1

Descriptive Statistics, Experiments 1A and 1B

\begin{tabular}{lccccccc}
\hline & \multicolumn{4}{c}{ No Responses } & & \multicolumn{3}{c}{ Yes Responses } \\
\cline { 2 - 4 } \cline { 6 - 7 } & Experimental & Neighbor & Control & Neighbor & & Animals & Plants \\
\hline Example & leotard & leopard & cellar & collar & & hamster & willow \\
Frequency & 10.2 & 16.3 & 9.9 & 33.7 & & 10.9 & 11.1 \\
Log-transformed frequency & 2.4 & 2.9 & 2.4 & 3.5 & & 2.5 & 2.5 \\
Length & 5.9 & 5.9 & 5.9 & 5.9 & & 5.8 & 5.9 \\
\hline
\end{tabular}

Note-The two sets of neighbors were never presented during the experiment. 

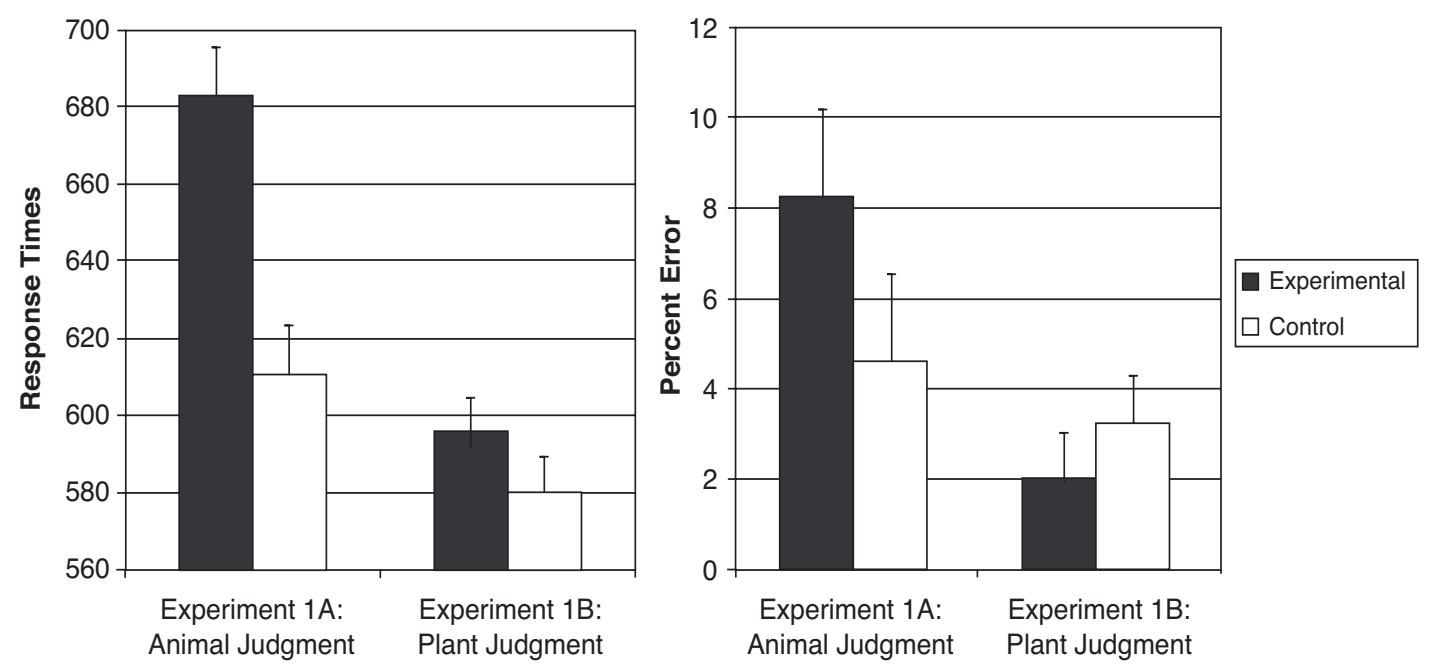

Figure 1. Response times and error rates for Experiments 1A and $1 \mathrm{~B}$.

$\left.2.3, p<.05 ; t_{2}(56)=1.1, p>.2\right]$. In contrast, $1.3 \%$ fewer errors were made to the experimental words. This difference was not significant (both $p \mathrm{~s}>.3$ ).

Experiments 1A and 1B: Combined analysis. Response times were subjected to analyses of variance with task (Experiment 1A vs. Experiment 1B) and neighbor type (experimental vs. control) as factors. Responses in Experiment 1A were slower than those in Experiment 1B. This difference was marginal in the participants analysis and was significant in the items analysis $\left[F_{1}(1,32)=3.2\right.$, $\left.p=.08 ; F_{2}(1,56)=39.3, p<.001\right]$. The main effect of neighbor type was significant: Responses to experimental words were slower than those to control words $\left[F_{1}(1,32)=\right.$ $\left.67.5, p<.001 ; F_{2}(1,56)=12.2, p<.001\right]$. Crucially, the interaction between these variables was significant $\left[F_{1}(1,32)=30.0, p<.001 ; F_{2}(1,56)=9.1, p<.005\right]$; the disadvantage for the words with animal neighbors was significantly greater in Experiment 1A.

The error rates showed a similar pattern: There were significantly more errors in Experiment $1 \mathrm{~A}$ than in Experiment $1 \mathrm{~B}\left[F_{1}(1,32)=7.2, p<.01 ; F_{2}(1,56)=15.4\right.$, $p<.001]$. The main effect of neighbor type was not significant $\left[F_{1}(1,32)=0.4, p=.5 ; F_{2}(1,56)=1.1, p=.3\right]$. Again, the interaction between these variables was significant $\left[F_{1}(1,32)=4.9, p<.05 ; F_{2}(1,56)=10.0, p<\right.$ $.005]$, so that the disadvantage for the words with animal neighbors was present only in Experiment 1A.

For the yes responses to category exemplars, the animals in Experiment 1A were responded to $19 \mathrm{msec}$ more slowly than the plants in Experiment 1B. This difference was not significant $\left[t_{1}(32)=0.6, p=.6 ; t_{2}(28)=1.5\right.$, $p=.16]$. In contrast, there were fewer errors for the animals in Experiment 1A (5.1\%) than for the plants in Experiment $1 \mathrm{~B}(7.1 \%)$. This difference was not significant $\left[t_{1}(32)=1.0, p=.3 ; t_{2}(28)=0.7, p=.5\right]$.

\section{Discussion}

These results confirm that it is difficult to decide that leotard is not an animal, because of its neighbor leop- ard. In Experiment 1A, the participants were $72 \mathrm{msec}$ slower for such words as leotard than for control words. In Experiment 1B, when the participants made decisions about plants, this difference was reduced to $15 \mathrm{msec}$. It is not clear whether this small residual difference (which was not significant in the items analysis) reflects accidental differences between the two sets of items, or whether it arises because animals and plants share some semantic features. This semantic overlap may cause interference from the animal neighbors on the plant decision. Whichever explanation is correct, the critical result is the significant interaction between task and condition: Animal neighbors interfere more in the animal decision task than in the plant decision task. This finding that when we read words, some aspect of their neighbors' meaning can be activated is consistent with the view that the meanings of written words begin to be activated before they are uniquely identified. ${ }^{1}$

There are, however, two alternative explanations of this effect. First, it may be driven by semantic-associative relationships between items in the list, rather than the relationship between the items and the named categories. For example, puppy (category exemplar in Experiment 1A) has a strong semantic-associative relationship to kitten (neighbor of the experimental item mitten). Therefore, when participants see puppy, the lexical entry of kitten may be automatically activated because of this relationship, and priming from this lexical entry may interfere with the later recognition of mitten. Under this account, the effect arises because of kitten's relationship to puppy, and not because kitten is a member of the category ani$\mathrm{mal}$, and it does not require that there is cascaded activation of semantics. Although this account is possible, it seems unlikely for several reasons. First, the temporal gap between such pairs was relatively long and highly variable. Furthermore, there are relatively few pairs that have such strong associations. Published association norms are available for $90 \%$ of the category exemplars (Kiss, Armstrong, Milroy, \& Piper, 1973; Moss \& Older, 
1996; Nelson, McEvoy, \& Schreiber, 1999), and these were used to estimate the possible contribution of relationships between individual items in the experiment. For each animal neighbor (e.g., kitten), the category exemplar with the highest associative strength to that neighbor was selected (e.g., puppy). The mean associative strength of these pairs was only $3.8 \%$ (i.e., $3.8 \%$ of participants generate the neighbor in response to the category exemplar). Therefore, the probability of participants spontaneously generating sufficient animal neighbors to produce robust priming is low. However, since there are some pairs with relatively high associative strength (e.g., spider-fly, 18\%), the combined items analysis was repeated without the items whose associative strength is greater than $5 \% .^{2}$ The critical interaction between task and neighbor type remained significant $[F(1,40)=4.9$, $p<.05]$. This suggests that the effect occurs because of cascaded activation of semantic features, and not because of semantic-associative priming effects.

A second possibility is that this effect should be interpreted within the form-first model proposed by Forster and Hector (2002) to account for the turple effect. Rather than assuming that some aspect of leopard's semantic representation is accessed in response to leotard, Forster and Hector would argue that the lexical representation of leopard contains sufficient semantic information to interfere with the task. Although the turple and leotard effects are consistent with both these accounts, Forster and Hector suggested that their finding that participants are slower to categorize low-frequency words as nonanimals than high-frequency words ${ }^{3}$ is inconsistent with models in which there is cascaded activation of semantics and no responses are generated when the participant reaches a deadline without activating any animal semantic features (e.g., Carreiras, Perea, \& Grainger, 1997). However, as was noted by Forster and Hector, this frequency effect can be explained within the cascaded framework if we assume that participants monitor the activation level of animal semantic features whose initial resting activation level is not zero. Animal words will turn these features on, whereas nonanimals will turn them off. Both of these processes are likely to be sensitive to frequency, and if we assume that no responses are made when the activation of these features approaches zero, this account can explain the frequency effect.

In summary, these data cannot discriminate between models in which the semantic representations of visually similar words are accessed in parallel and models in which lexical entries themselves contain some semantic information. However, although there are important differences between these accounts, they share the notion that semantic information becomes available before words are uniquely identified and, therefore, allow for the possibility that this semantic information may influence the discrimination between lexical neighbors. Future work looking at the circumstances in which there is semantic interference from lexical neighbors may allow us to determine which aspects of word meanings are activated before words have been uniquely recognized.
There is one further interesting aspect of this study. In Experiment 1A, the participants saw many animal names, and the task was an animal judgment. Therefore, the animal neighbors will have been primed both by the preceding animal names and by the expectancy created by the task. It is possible that without such contextual priming, there may be no semantic activation of neighbors. However, this does not detract from the conclusion that words' meanings can be activated in response to their neighbors. In normal reading, words are often highly primed by their context, and these are the conditions in which feedback from semantics could help a word win the race for recognition over inappropriate competitors. When words are not constrained by their context, information about their meaning is unlikely to improve recognition. Therefore, although this effect may be seen only under conditions in which the neighbor is being primed, this does not compromise the claim that the meanings of lexical neighbors can be activated.

So what are the implications of this result for the debate about whether there is feedback from semantics to form-based lexical representations? Although this result clearly does not show that there is feedback, in conjunction with the data from Forster and Hector (2002), it does show that some aspect of the meanings of multiple candidate words can be activated in parallel before a word is uniquely recognized. Therefore, semantic information is available at a point in time at which it might help to discriminate between visually similar words. Further studies are needed to determine the nature of the semantic information that is retrieved, and whether this information does indeed influence our ability to recognize words.

\section{REFERENCES}

Batyen, R. H., Piepenbrock, R., \& Gulikers, L. (1995). The CELEX lexical database [CD-ROM]. Philadelphia: University of Pennsylvania, Linguistic Data Consortium.

Balota, D. A., Ferraro, F. R., \& Connor, L. T. (1991). On the early influence of meaning in word recognition: A review of the literature. In P. J. Schwanenflugel (Ed.), The psychology of word meanings (pp. 187-222). Hillsdale, NJ: Erlbaum.

Carreiras, M., Perea, M., \& Grainger, J. (1997). Effects of orthographic neighborhood in visual word recognition: Cross-task comparisons. Journal of Experimental Psychology: Learning, Memory, \& Cognition, 23, 857-871.

Coltheart, M., DavelaAr, E., Jonasson, J. T., \& Besner, D. (1977). Access to the internal lexicon. In S. Dornic (Ed.), Attention and performance VI (pp. 535-555). Hillsdale, NJ: Erlbaum.

Coltheart, M., Rastle, K., Perry, C., Ziegler, J., \& Langdon, R. (2001). DRC: A dual route cascaded model of visual word recognition and reading aloud. Psychological Review, 108, 204-256.

Forster, K. I. (1976). Accessing the mental lexicon. In R. J. Wales \& E. Walker (Eds.), New approaches to language mechanisms (pp. 257287). Amsterdam: North-Holland.

ForSTER, K. I. (1981). Priming and the effects of sentence and lexical contexts on naming time: Evidence for autonomous lexical processing. Quarterly Journal of Experimental Psychology, 33A, 465-495.

Forster, K. I., \& Forster, J. C. (2003). DMDX: A Windows display program with millisecond accuracy. Behavior Research Methods, Instruments, \& Computers, 35, 116-124.

Forster, K. I., \& HECTOR, J. (2002). Cascaded versus noncascaded models of lexical and semantic processing: The turple effect. $\mathrm{Mem}-$ ory \& Cognition, 30, 1106-1117.

GASKELL, M. G., \& MARSLEN-WILSON, W. D. (1997). Integrating form 
and meaning: A distributed model of speech perception. Language \& Cognitive Processes, 12, 613-656.

Kiss, G. R., Armstrong, C., Milroy, R., \& PiPer, J. (1973). An associative thesaurus of English and its computer analysis. In A. J. Aitkin, R. W. Bailey, \& N. Hamilton-Smith (Eds.), The computer and literary studies (pp. 153-165). Edinburgh: University Press.

Marslen-WiLson, W. D. (1973). Linguistic structure and speech shadowing at very short latencies. Nature, 244, 522-523.

Marslen-Wilson, W. D. (1985). Speech shadowing and speech comprehension. Speech Communication, 4, 55-73.

Marslen-WiLson, W. D. (1987). Functional parallelism in spoken word-recognition. Cognition, 25, 71-102.

Moss, H., \& OLDER, L. (1996). Birkbeck word association norms. Hove, U.K.: Psychology Press.

Nelson, D. L., McEvoy, C. L., \& Schreiber, T. A. (1999). The University of South Florida word association, rhyme, and word fragment norms [On line]. Available at w3.usf.edu/FreeAssociation/.

Plaut, D. C., McClelland, J. L., Seidenberg, M. S., \& Patterson, K. (1996). Understanding normal and impaired word reading: Computational principles in quasi-regular domains. Psychological Review, 103, 56-115.
Tyler, L. K., Voice, J. K., \& Moss, H. E. (2000). The interaction of meaning and sound in spoken word recognition. Psychonomic Bulletin \& Review, 7, 320-326.

Winer, B. J., Brown, D. R., \& Michels, K. M. (1991). Statistical principles in experimental design (3rd ed.). New York: McGraw-Hill.

Zwitserlood, P. (1989). The locus of effects of sentential-semantic context in spoken-word processing. Cognition, 32, 25-64.

\section{NOTES}

1. Note that these results do not show that there is no competition from neighbors that are not category members, but only that any such competition is reduced, as compared with that produced by category members.

2. These items were chimp, fly, hawk, horse, kitten, moth, raven, and shrimp.

3. This frequency effect was replicated in the control items in the experiments reported here. There is a significant correlation between the log-transformed lemma frequency of these items and the response times in both Experiment $1 \mathrm{~A}[r(29)=.50, p<.01]$ and Experiment $1 \mathrm{~B}$ $[r(29)=.49, p<.01]$.

APPENDIX

Experiments 1A and 1B: Stimuli

\begin{tabular}{|c|c|c|c|c|c|}
\hline \multicolumn{4}{|c|}{ No Responses } & \multicolumn{2}{|c|}{ Yes Responses } \\
\hline Experimental & $\begin{array}{c}\text { Animal } \\
\text { Neighbor }\end{array}$ & Control & $\begin{array}{c}\text { Nonanimal } \\
\text { Neighbor }\end{array}$ & $\begin{array}{l}\text { Experiment 1A: } \\
\text { Animals }\end{array}$ & $\begin{array}{c}\text { Experiment 1B: } \\
\text { Plants }\end{array}$ \\
\hline flu & fly & imp & amp & bee & oak \\
\hline plea & flea & neon & noon & toad & plum \\
\hline myth & moth & poem & poet & vole & lily \\
\hline gawk & hawk & ogle & ogre & crow & lime \\
\hline crib & $\mathrm{crab}$ & noun & noon & wasp & pine \\
\hline skulk & skunk & preen & green & rhino & mango \\
\hline chirp & chimp & shunt & stunt & gecko & tulip \\
\hline shred & shrew & quest & guest & puppy & birch \\
\hline haven & raven & taboo & tabor & prawn & shrub \\
\hline utter & otter & aloud & cloud & eagle & olive \\
\hline cameo & camel & elope & slope & trout & daisy \\
\hline horde & horse & scold & scald & bear & grass \\
\hline combat & wombat & random & ransom & budgie & radish \\
\hline muffin & puffin & garret & garnet & cuckoo & orchid \\
\hline thrash & thrush & crunch & crutch & weasel & poplar \\
\hline weaver & beaver & beacon & deacon & dolphin & turnip \\
\hline scrimp & shrimp & grouch & crouch & spider & celery \\
\hline wizard & lizard & hermit & permit & beetle & banana \\
\hline mitten & kitten & toffee & coffee & donkey & willow \\
\hline saloon & salmon & cellar & collar & monkey & orange \\
\hline mobster & lobster & glazier & glacier & raccoon & cypress \\
\hline culture & vulture & product & produce & penguin & apricot \\
\hline leotard & leopard & gunnery & nunnery & sparrow & heather \\
\hline shallow & swallow & scarlet & starlet & hamster & blossom \\
\hline thicken & chicken & auction & suction & insect & cabbage \\
\hline woodwork & woodworm & blockade & blockage & cheetah & daffodil \\
\hline sterling & starling & friction & fraction & kangaroo & cucumber \\
\hline pleasant & pheasant & laughter & daughter & mosquito & chestnut \\
\hline earthwork & earthworm & milestone & millstone & butterfly & buttercup \\
\hline
\end{tabular}

Note-The two sets of neighbors were never presented during the experiment. 